\title{
Infusão de Insulina Subcutânea Contínua em Gestante com Diabetes Tipo 1: Relato de Caso e Revisão da Literatura
}

\begin{abstract}
RESUMO
A gestação em portadoras de diabetes tipo 1 é alvo de múltiplas tentativas para o bom controle glicêmico desde o período pré-concepcional, com a terapia com infusão de insulina subcutânea contínua apresentando opção terapêutica adicional na tentativa de alcançar as metas glicêmicas. Apresentamos o caso de uma paciente com diabetes tipo 1, não controlada com múltiplas injeções diárias, cuja instalação da bomba de insulina ocorreu durante o segundo trimestre de gestação, com sucesso terapêutico. Arq Bras Endocrinol Metab 2008; 52/4:697-700)
\end{abstract}

Descritores: Bomba de insulina; Gestação; Diabetes tipo 1.

\section{apresentação}

de caso

JANICE SepÚlVeda ReIS

RODRIGO NUNES LAMOUNIER

PatrícIa A. F. C. Menezes

Maria R. Calsolari

SAULO PURISCH

Serviço de Endocrinologia e Metabologia da Santa Casa de Belo Horizonte, MG, Brasil. \section{nancy: Case Report and Literature Review.}

Continuous Subcutaneous Insulin Infusion in type 1 diabetic during preg-

The type 1 diabetic patient pregnancy is subjected to various attempts to obtain good glycemic control, since the pre-conception period throughout the gestation. Continuous subcutaneous insulin infusion (CSII) is a therapeutic tool to achieve the glycemic targets. Here it is presented the case of a Type 1 diabetic woman, who was not under multiple insulin daily injections therapy, and whose insulin therapy started on CSII during the second trimester of gestation, obtaining successful therapeutic outcome. Arq Bras Endocrinol Metab 2008; 52/4:697-700)

Keywords: Insulin pump; Pregnancy; Type 1 diabetes.
DIABETES MELITO ESTÁ ASSOCIADO com o aumento no risco para morbimortalidade materna e fetal, sendo a principal meta do tratamento da gestante diabética a manutenção dos níveis glicêmicos dentro da normalidade, especialmente no primeiro trimestre $(1,2)$. O diabetes tipo 1 , outro grande desafio para o especialista, torna a gravidez alvo de múltiplas tentativas para o bom controle glicêmico, desde o período pré-concepcional (3). A terapia com infusão de insulina subcutânea contínua (CSII/bomba de insulina), ainda pouco indicada em nosso meio, constitui opção terapêutica adicional na tentativa de se alcançar metas glicêmicas durante a gestação, em casos selecionados.

Apresentamos o caso de uma paciente portadora de diabetes tipo 1, cuja instalação da CSII ocorreu durante o segundo trimestre de gestação, com sucesso terapêutico.
Recebido em 14/05/2007 Aceito em 09/01/2008 


\section{RELATO DE CASO}

Paciente com 22 anos de idade, diabética tipo 1 desde os 11 anos, de difícil controle e com múltiplas internações por cetoacidose diabética (CAD), internada em CAD, sendo diagnosticada gestação de quatro semanas. Foi iniciado acompanhamento no ambulatório de diabetes tipo 1 da Santa Casa de Belo Horizonte com 18 semanas de gestação, apresentando ultra-som obstétrico sem anormalidades, perfil glicêmico lábil, hipoglicemias graves freqüentes $(<40 \mathrm{mg} / \mathrm{dL})$, hemoglobina glicada (HbAlc) de 13,9\%, com múltiplas tentativas de ajuste nas doses de insulina, conforme relatório médico. Relatava seguir corretamente as orientações nutricionais (estava em contagem de carboidratos há dois anos), aplicações corretas das insulinas NPH (3 doses/dia) e lispro (4 refeições/dia), com esquemas de correções de hiperglicemias (dose diária total de insulina: 0,9 U/kg/ dia). Optou-se por instalação da CSII (Medtronic MiniMed 508), com insulina lispro, realizada com 21 semanas de gestação. A paciente evoluiu com grande melhora no perfil glicêmico, com hipoglicemias episódicas (sempre acima de $50 \mathrm{mg} / \mathrm{dL}$ ), mantendo-se dentro das metas para gestação, com a evolução nos exames laboratoriais apresentados na Tabela 1. Entrou em trabalho de parto com 36 semanas de idade gestacional, submetida a parto normal, sem intercorrências, sem relatos de hipoglicemia neonatal, com feto dentro dos percentis da normalidade, sem malformações (APGAR 9/9; peso e comprimento ao nascer: $3,150 \mathrm{~kg}$ e 49 $\mathrm{cm})$. Optamos por manutenção do CSII no pós-parto, com a paciente mantendo-se satisfeita com a nova tera- pêutica, com consultas regulares, sem novas internações, com controle glicêmico satisfatório. Médias glicêmicas quatro semanas antes e após a instalação da CSII são apresentadas na Figura 1.

Tabela 1. Exames laboratoriais no seguimento.

\begin{tabular}{ccccc}
\hline $\begin{array}{c}\text { Idade } \\
\text { gestacional }\end{array}$ & $\begin{array}{c}\mathbf{1 8} \\
\text { semanas }\end{array}$ & $\begin{array}{c}\mathbf{2 1} \\
\text { semanas }\end{array}$ & $\begin{array}{c}\mathbf{3 0 . 6} \\
\text { semanas }\end{array}$ & $\begin{array}{c}\text { Pós-parto } \\
\text { imediato }\end{array}$ \\
\hline $\begin{array}{c}\text { Frutosamina } \\
\mathrm{HbAlc}\end{array}$ & $\mathbf{4 5 8}$ & & 208,30 & 190 \\
\hline $\begin{array}{c}\text { Clearence } \\
\text { de creatinina } \\
\left(\mathrm{mL} / \mathrm{min}^{2} \mathrm{~m}^{2}\right)\end{array}$ & $17 \%$ & & $5,4 \%$ & $6,4 \%$ \\
\hline $\begin{array}{c}\text { Proteinúria } \\
24 \text { horas }\end{array}$ & $0,210 \mathrm{~g}$ & $0,102 \mathrm{~g}$ & \\
\hline
\end{tabular}

\section{DISCUSSÃo}

Historicamente, portadoras de diabetes tipo 1 eram desencorajadas a engravidar por causa do alto risco de complicações maternas e fetais. Nas últimas décadas, com o melhor entendimento do manejo da doença no período pré-gestacional e na gravidez, somados aos avanços terapêuticos, modificou-se este paradigma.

Com metas glicêmicas durante a gestação inferiores ao período pré-concepção, torna-se difícil o sucesso terapêutico com tratamento convencional, sendo indicado tratamento intensivo com múltiplas injeções diárias
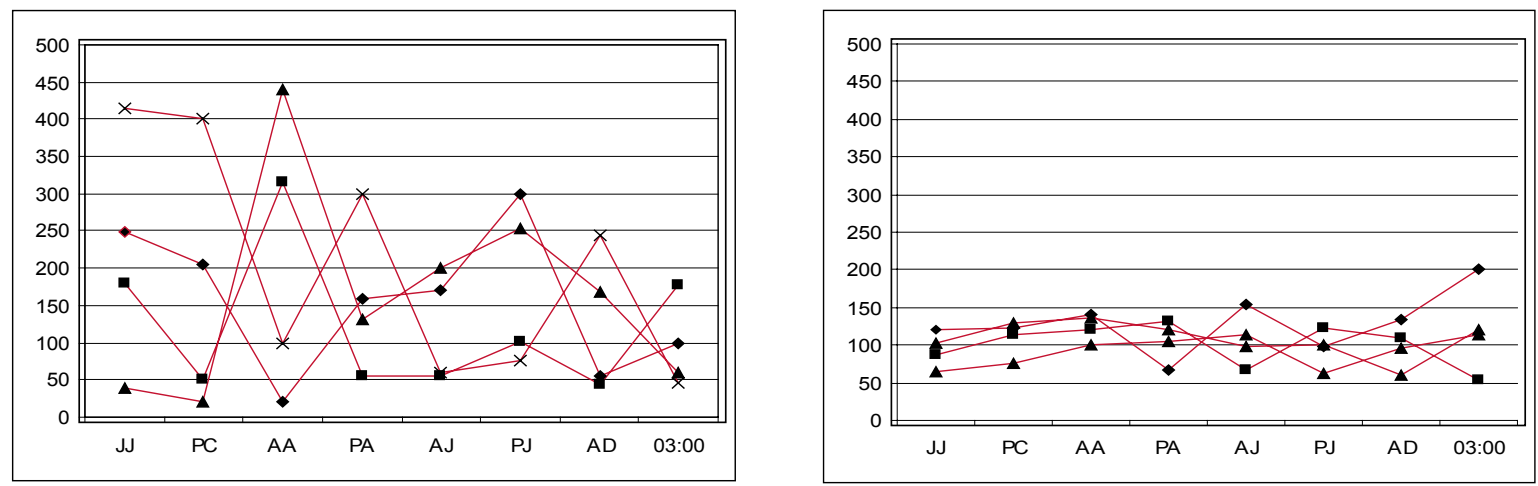

JJ: jejum; PC: 2 horas após o café; AA: antes do almoço; PA: 2 horas após o almoço; AJ: antes do jantar; PJ: 2 horas após o jantar; AD: ao deitar.

Figura 1. Médias glicêmicas quatro semanas antes e pós-CSII. 
(MID) ou CSII, além de orientações nutricionais adaptadas para esta fase e monitorização glicêmica mais rigorosa (4), o que preferencialmente devem ser otimizados pelo menos três meses antes da concepção.

A experiência com a terapia com bomba de insulina tem aumentado nas últimas três décadas, sendo atualmente potenciais indicações para seu uso: hipoglicemias graves recorrentes, amplas flutuações nos níveis glicêmicos, HbAlc fora das metas com MID, complicações microvasculares e/ou fatores de risco para complicações macrovasculares, comprometimento do estilo de vida com MID e gestação com controle glicêmico insatisfatório (idealmente pré-concepção), entre outras, inclusive a opção do paciente por esta forma de tratamento. Os principais estudos com diabéticos tipo 1 adultos, excluindo-se gestantes, sugerem forte evidência de que a terapia com CSII seja segura e esteja associada com significante melhora do controle glicêmico (5-7). Os estudos envolvendo gestantes e CSII são limitados e envolvem pequeno número de participantes.

Estudos iniciados no período pré-concepção ou no primeiro trimestre da gestação, não envolvendo pacientes com diabetes hiperlábil, com HbAlc comparáveis e classificação de White, demonstraram que o controle glicêmico e o prognóstico fetal em gestantes com diabetes tipo 1 foram similares entre os dois regimes de tratamento intensivo, não tendo sido encontradas diferenças entre os grupos em relação ao número de hospitalizações ou na mortalidade perinatal, na glicohemoglobina, na dose de insulina, no peso ao longo da gestação, na taxa de malformações congênitas ou nas complicações maternas (pré-eclâmpsia, hipertensão, cetoacidose, hipoglicemias) e fetais (hipoglicemia, hipocalcemia, hiperbilirrubinemia, asfixia etc.), sugerindo que a CSII deveria ser reservada aos casos de diabetes de difícil controle, em que a possibilidade de diferentes taxas de infusão basal ao longo do dia diminuiria os episódios de hipoglicemias e flutuações glicêmicas, facilitando ainda o manejo do período de hiperemese e o controle glicêmico durante e após o parto (8-12).

Zoric e cols. (13), após instalação de CSII em 17 diabéticas tipo 1 com diagnóstico recente de gravidez e sem controle glicêmico, com acompanhamento durante o primeiro trimestre da gestação, observaram melhora importante na $\mathrm{HbAlc}(8,94 \pm 1,62$ versus $6,9 \pm 1,22$ $\%, \mathrm{p}<0,05)$, na média glicêmica durante monitorização domiciliar $(9,23 \pm 2,2$ versus $6,41 \pm 1,72 \mathrm{mmol} / 1$, $\mathrm{p}<0,01)$ e na dose diária total de insulina $(0,66 \pm 0,22$ versus $0,55 \pm 0,13 \mathrm{U} / \mathrm{kg} /$ dia $)$ durante a terapia, correlacionando-os inversamente $(\mathrm{r}=-0,6, \mathrm{p}<0,05)$ com o peso fetal e o escore de APGAR.

Gabbe e cols. (14), analisando os benefícios, os riscos e a satisfação de diabéticas tipo 1 associados com o uso da bomba de insulina, compararam pacientes que iniciaram terapia com bomba em torno de 16,8 semanas de gestação $(\mathrm{n}=24)$ com aquelas tratadas com MID ( $\mathrm{n}=24)$ ou que já engravidaram em uso da CSII $(\mathrm{n}=12)$ e observaram que não houve piora do controle glicêmico com instalação da bomba durante a gestação, sendo comparáveis entre os três grupos os benefícios (HbAlc e sucesso na gravidez) durante o tratamento CSII $\times$ MID, com manutenção da bomba como terapia de escolha no pós-parto.

Os pré-requisitos para a escolha da terapia com bomba de insulina incluem, principalmente, a capacidade para manusear a bomba, contar carboidratos e a disposição para monitorar e registrar glicemias, levando-se em consideração que a dose total diária de insulina atende melhor às gestantes quando divididas em $40 \%$ como basal e $60 \%$ nos bólus alimentares $(15)$, retornando à proporção $50 \%$ basal: $50 \%$ bólus no pós-parto (16).

O controle glicêmico satisfatório, independentemente da modalidade de tratamento, é o principal influenciador do prognóstico materno e fetal na gestação de pacientes diabéticas tipo 1 , com o aconselhamento pré-gestacional e o tratamento intensivo com insulina, sendo as principais opções para alcançá-lo. Embora a CSII não tenha se mostrado superior a MID durante a gestação nas pacientes com controle adequado, deve-se considerar que a principal indicação para a bomba de insulina em diabéticas grávidas seja o mau controle glicêmico com MID. Portanto, a terapia com infusão contínua de insulina deve ser considerada como opção terapêutica no acompanhamento de gestantes com diabetes tipo 1, nos casos de difícil controle, que coloque em risco o sucesso da gestação, mostrando-se segura mesmo quando iniciada durante este período.

\section{REFERÊNCIAS}

1. Schmidt MI, Reichelt AJ, pelo Grupo de Estudo em Diabetes e Gravidez. Consenso sobre diabetes gestacional e diabetes pré-gestacional. Arq Bras Endocrinol Metab. 1999;43:14-8.

2. Evers IM, de Valk HW, Visser GH. Risk of complications of pregnancy in women with type 1 diabetes: nationwide prospective study in the Netherlands. BMJ. 2004;328:915.

3. Kitzmiller JL, Gavin LA, Gin GD, Jovanovic-Peterson L, Main EK, Zigrang WD. Preconception care of diabetes: glycemic 
control prevents congenital anomalies. JAMA. 1991;265: 731-6.

4. The Diabetes Control and Complications Trial Research Group. The effect of intensive treatment of diabetes on the development and progression of long-term complications in insulindependent diabetes mellitus. N Engl J Med. 1993;329:977-86

5. Weissberg-Benchell J, Antisdel-Lomaglio J, Seshadri R. Insulin pump therapy: a meta-analysis. Diabetes Care. 2003;26 (4):1079-87.

6. Pickup JC, Kidd J, Burmiston S, Yemane N. Long-term continuous subcutaneous insulin infusion in diabetics at home. Lancet. 1979;2(8148):870-3.

7. Bode BW, Steed RD, Davidson PC. Reduction in severe hypoglycemia with long-term continuous subcutaneous insulin infusion in type I diabetes. Diabetes Care. 1996;19(4):324-7.

8. Leveno KJ, Fortunato SJ, Raskin P, Williams ML, Whalley PJ. Continuous subcutaneous insulin infusion during pregnancy. Diabetes Res Clin Pract. 1988;4(4):257-68.

9. Gimenez M, Conget I, Nicolau J, Pericot A, Levy I. Outcome of pregnancy in women with type 1 diabetes intensively treated with continuous subcutaneous insulin infusion or conventional therapy. A case-control study. Acta Diabetol. 2007;44(1): 34-7.

10. Lapolla A, Dalfra MG, Masin M, Bruttomesso D, Piva I, Crepaldi $C$, et al. Analysis of outcome of pregnancy in type 1 diabetics treated with insulin pump or conventional insulin therapy. Acta Diabetol. 2003;40(3):143-9.

11. Hieronimus S, Cupelli C, Bongain A, Durand-Reville M, Berthier F, Fenichel P. Pregnancy in type 1 diabetes: insulin pump versus intensified conventional therapy. Gynecol Obstet Fertil. 2005;33(6):389-94.
12. Gottilieb PA, Frias JP, Peters KA, Chillara B, Garg SK. Optimizing insulin therapy in pregnant women with type 1 diabetes mellitus. Treat Endocrinol, 2002;1(4):235-40.

13. Zoric S, Micic D, Kendereski A, Sumarac-Dumanovic M, Cvijovic $G$, Pejkovic D, et al. Use of continuous subcutaneous insulin infusion by a portable insulin pump during pregnancy in women with type 1 diabetes mellitus. Vojnosanit Pregl. 2006;63(7):648-51.

14. Gabbe SG, Holing E, Temple P, Brown ZA. Benefits, risks, costs, and patient satisfaction associated with insulin pump therapy for the pregnancy complicated by type 1 diabetes mellitus. Am J Obstet Gynecol. 2000;182(6):1283-91.

15. Rudolf MC, Coustan DR, Sherwin RS, Bates SE, Felig P, Genel $M$, et al. Efficacy of the insulin pump in the home treatment of pregnant diabetics. Diabetes, 1981;30(11):891-5.

16. Davidson PC. Bolus and supplement insulin. In: Fredrickson L, editor. The insulin pump therapy book: insights from the experts. Sylmar, Ca: Minimed; 1995. p. 59-71.

Endereço para correspondência:

Janice Sepúlveda Reis

CEPCEM

Av. Francisco Sales, $1111,5^{\circ}$ andar, ala D, Santa Efigênia

30150-221 - Belo Horizonte, MG

E-mail: janicesepulveda@terra.com.br 